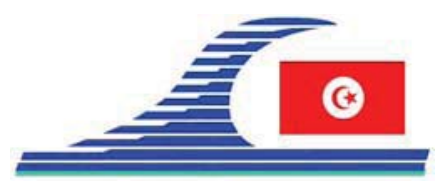

Conférence Méditerranéenne Côtière et Maritime EDITION 1, HAMMAMET, TUNISIE (2009)

Coastal and Maritime Mediterranean Conference

Disponible en ligne - http://www.paralia.fr-Available online

\title{
Système temps réel de prévision du devenir des rejets en mer
}

\author{
Philippe CRANEGUY ${ }^{1}$, Alain COAT ${ }^{1}$, Vincent MARIETTE ${ }^{1}$, \\ Loic CROISSANT ${ }^{2}$, Philippe VENCHIARUTTI ${ }^{2}$, Dominique VASSELIN ${ }^{2}$
}

1. Actimar, 36 quai de la Douane, 29200 Brest, France. craneguy@actimar.fr; coat@actimar.fr;mariette@actimar.fr

2. SAUR, ZA porte Océane, rue du Portugal, 56400 Auray, France. lcroissa@saur.fr ; pvenchia@saur.fr

\section{Résumé :}

Dans le cadre du projet Pôle Mer Previcot (www.previcot.com), Actimar a développé un système opérationnel de prévision à haute résolution de la qualité des eaux littorales. Ce système, validé dans le cadre du rejeu de l'été 2008, est opéré depuis juin 2009 sur un site pilote du littoral français en partenariat avec la SAUR. L'analyse en cours des performances du système opérationnel vise à définir les actions de recherche permettant d'améliorer le service opérationnel.

\section{Mots-clés :}

Environnement littoral - Qualité des eaux littorales - Système opérationnel

\section{Introduction}

Le suivi de rejets en mer provenant d'émissaires de stations d'épuration, d'installations industrielles en milieu littoral ou encore du réseau d'eaux pluviales revêt souvent un caractère déterminant pour la qualité des eaux littorales. Cette note présente le système opérationnel de prévision à très haute résolution de dérive et de diffusion de ces panaches développé par Actimar dans le cadre du projet pôle mer Previcot (www.previcot.com) et opéré depuis juin 2009 sur un site pilote du littoral français.

\section{Système opérationnel de prévision de la qualité des eaux littorales}

\subsection{Conception du système opérationnel}

Le démonstrateur mis en place par Actimar vise à répondre aux besoins des conchyliculteurs et des collectivités territoriales en terme de suivi et de prévision de qualité des eaux. Le système opérationnel est mis en place en Baie de Quiberon (Bretagne Sud), qui est l'une des principales zones d'exploitation ostréicole de Bretagne et il peut aisément être mis en place sur d'autres zones d'intérêt.

Les produits développés dans le cadre de ce démonstrateur portent sur :

DOI: $10.5150 / \mathrm{cmcm} .2009 .059-\mathrm{Y}$ 
a) le suivi et la diffusion de rejets en mer : le système opérationnel de prévision météorologique, de prévision des panaches littoraux et de prévision de la qualité des eaux vise à permettre aux conchyliculteurs d'anticiper les situations à risque ;

b) la recherche de l'origine des pollutions : le système possède également un intérêt diagnostic à travers la recherche des origines et des causes des phénomènes de pollution. Il doit permettre d'inciter des aménagements ou des modes de gestion des rejets en mer ;

c) la prévision des conditions environnementales : les conditions météo-océaniques sont diffusées aux ostréiculteurs à fins de planification de leurs activités et d'anticipation des situations à risque : la température de fond constitue le paramètre le plus souvent impliqué dans les évènements de mortalité des huîtres (SOLETCHNIK et al., 2007).

\subsection{Spécification du système opérationnel}

Le système de prévision fonctionne en mode automatique, il prévoit de manière régulière les conditions environnementales et la qualité des eaux littorales, évalue les performances des produits par comparaison avec les observations disponibles. Ces informations sont accessibles aux utilisateurs sur un site internet.

Le système opérationnel est composé des éléments suivants :

a) un emboîtement de modèles météorologiques WRF (Weather Research and Forecasting Model), forcés par les champs NCEP-GFS, qui prédit sur un maillage fin de la zone d'étude (résolution spatiale de $2.7 \mathrm{~km}$ ) le vent et la pluviométrie ;

b) un système terrestre MIKE21 opéré par la SAUR intégrant des modèles hydrologiques des bassins versants, l'écoulement dans les réseaux d'eaux usées et pluviales, ainsi que les apports diffus, qui a pour fonction de prévoir les apports polluants (débit et teneurs) s'effectuant en mer ;

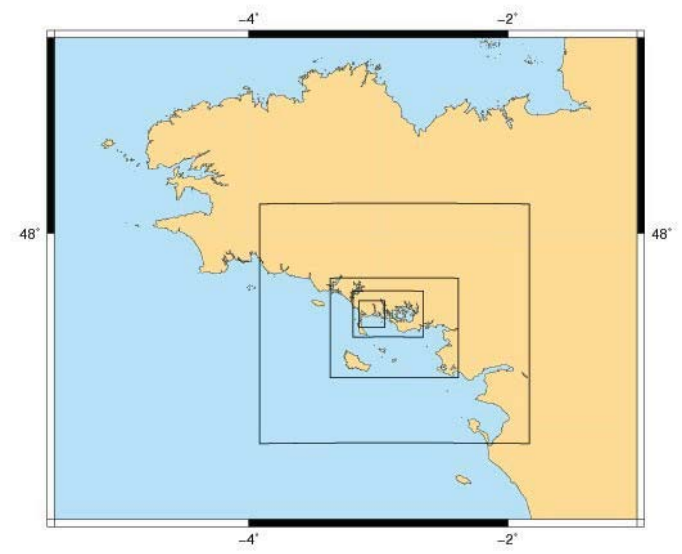

Figure 1. Emprise des emboîtements gigogne de modèles hydrodynamiques de résolution spatiale respective : $1300 \mathrm{~m}, 300 \mathrm{~m}, 100 \mathrm{~m}, 20 \mathrm{~m}$ sur l'horizontale et comprenant 12 niveaux sigma sur la verticale. 
c) un emboîtement de modèles hydrodynamiques tridimensionnels MARS (LAZURE \& DUMAS, 2008) prédisant également la dispersion des rejets dans le système à haute résolution (voir figure 1). Le modèle de grande emprise du système exploite pour conditions aux limites les prévisions issues du modèle opérationnel Manche-Gascogne de Previmer (www.previmer.fr).

Ces différents modèles sont intégrés pour produire de manière automatique une prévision des rejets, de la qualité des eaux et des conditions environnementales en Baie de Quiberon. Les performances du système de prévision sont évaluées par des mesures in situ qui fournissent en temps réel les paramètres météorologiques (vent, pluie, température de l'air), la salinité et la température de surface.

\subsection{Validation du système opérationnel}

Préalablement à la phase de démonstration opérationnelle, les différentes composantes du système opérationnel ont été calibrées et validées par comparaison avec les mesures disponibles dans la zone d'étude.

a) Validation de la composante météo-océanique :

Les performances des différents emboîtements de modèles météorologiques et hydrodynamiques ont été évaluées en Baie de Quiberon par comparaison aux données de vent, de pluie, de courants et en hydrologie. La figure 2 montre que la salinité de surface prévue par le système est en bon accord avec les mesures de salinité.

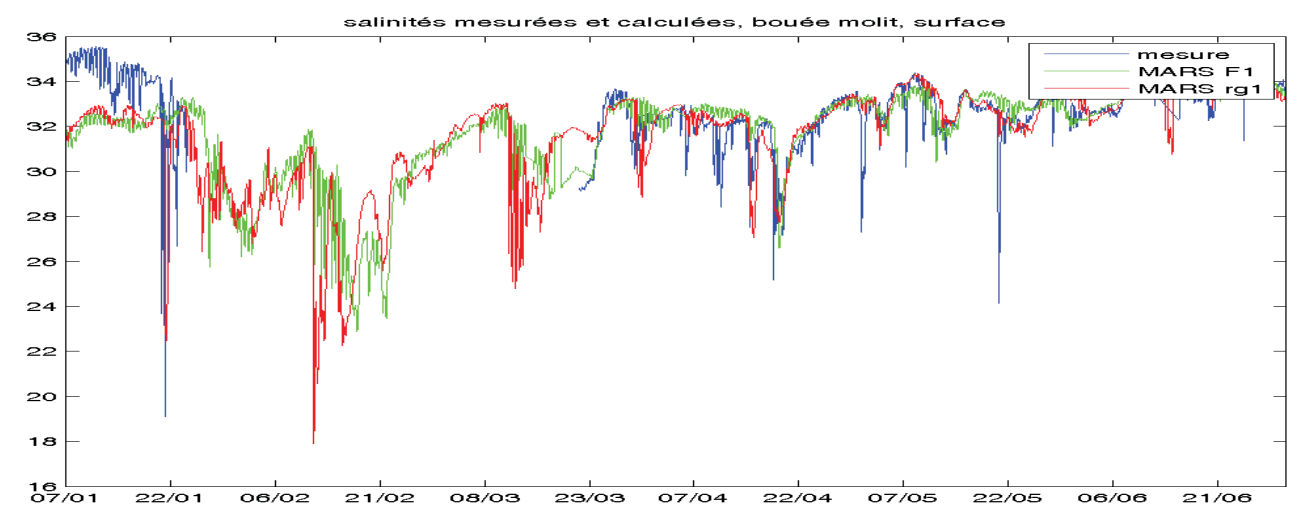

Figure 2. Validation en salinité : les évolutions temporelles des prévisions de salinité de surface (unité : psu) issues du modèle Previmer (en rouge) et du modèle emboîté (résolution de $1.3 \mathrm{~km}$, en vert) sont comparées aux mesures in situ (en bleu).

\section{b) Validation du module de dérive :}

Le module de dérive a été mis au point et sa validation a été menée dans le cadre d'un rejeu d'une situation mesurée à l'été 2008. Les rejets exploités lors du rejeu sont les rejets mesurés (débit et concentration) en différents points de la zone d'étude (station d'épuration et rejet pluvial). Le devenir des panaches est en bon accord avec les mesures lors des fortes concentrations en Escherichia Coli, qui apparaissent après un 
évènement pluvieux (non présenté). Pour des concentrations plus faibles, la concordance modèle-mesure diminue mais, tenant compte de la précision des mesures, les performances du système de prévision sont difficiles à évaluer dans ces situations.

\subsection{Démonstration opérationnelle}

Le système de prévision de la qualité des eaux est opérationnel depuis fin juin 2009. Les prévisions des conditions météo-océaniques et de la qualité des eaux littorales sont consultables sur un site internet à accès restreint (voir figure 3). Ces informations sont exploitées par les services techniques de la Ville pour anticiper les risques potentiels de pollution bactériologique et sont intégrées à leur plan de gestion de la qualité des eaux. L'analyse en cours des performances du système exploite un ensemble de données météo-océaniques et de qualité des eaux récoltées de juillet à septembre 2009. Elle vise à définir les actions de recherche qui permettront d'améliorer le service opérationnel.

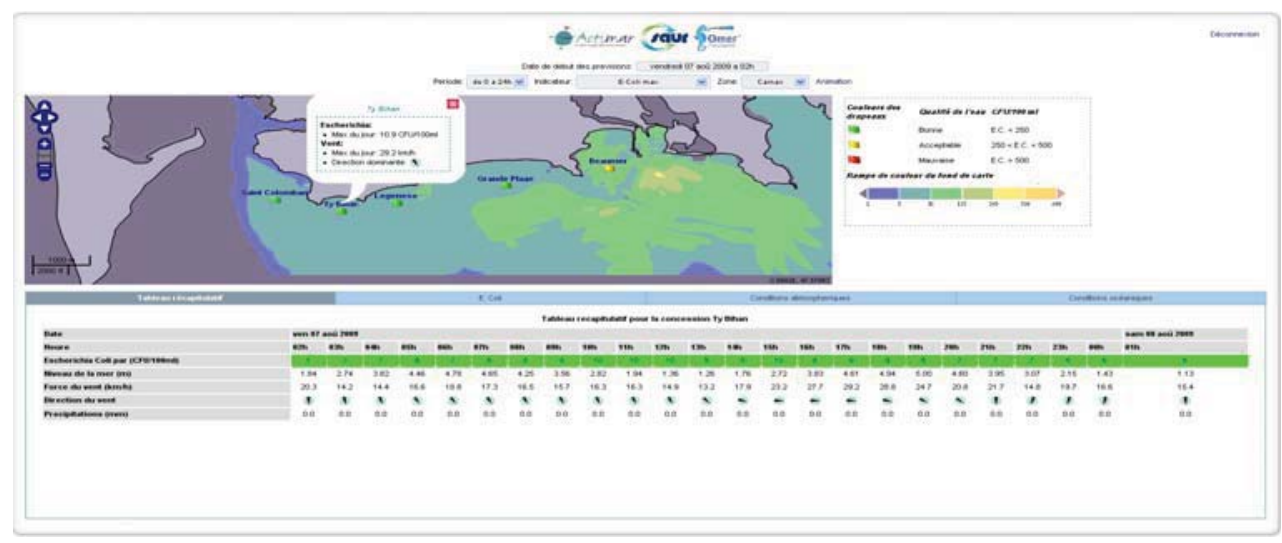

Figure 3. Site internet de prévision de la qualité des eaux littorales.

\section{Conclusions}

Actimar a développé et mis en place un système opérationnel de prévision de la qualité des eaux littorales. Le système, validé dans le cadre du rejeu de l'été 2008, est opéré depuis juin 2009 sur un site du littoral atlantique français en partenariat avec la SAUR. L'analyse en cours des performances du système vise à définir les actions prioritaires de recherche qui permettront d'améliorer le service opérationnel.

\section{Références bibliographiques}

LAZURE P., DUMAS F. (2007). An external-internal mode coupling foor a 3D hydrodynamical model for application at regional scale (MARS). Advances in Water Resources, Volume 31, pp 233-250.

SOLETCHNIK P., ROPERT M., MAZURIE J., FLEURY P.G., LE COZ F. (2007). Relationships between oyster mortality patterns and environmental data from monitoring databases along the coasts of France, Aquaculture, Volume 271, pp 384400 . 